\title{
Atitudes e Percepções Relativas ao Tratamento de Manutenção com Opiáceos em Portugal: Um Inquérito a Médicos, Doentes e Utilizadores de Opiáceos
}

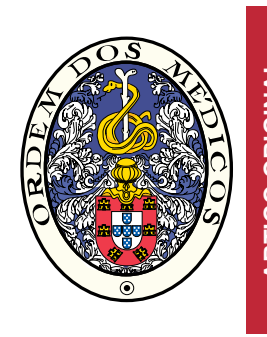

\author{
Attitudes and Beliefs Regarding Opioid Maintenance Treatment in Portugal: \\ A Survey to Physicians, Patients and Opioid Users \\ João GOULÃO 1 \\ Acta Med Port 2013 Sep-Oct;26(5):537-548
}

\section{RESUMO}

Introdução: Existem poucos estudos pan-europeus sobre programas terapêuticos com agonistas opiáceos, tanto da perspectiva dos prescritores, como dos doentes que os recebem e dos utilizadores de opióides que se mantêm fora do sistema de tratamento. $O$ projecto ACCESS, um estudo europeu realizado em 11 países, explorou as atitudes e percepções dos médicos, doentes e utilizadores de opióides quanto à qualidade e ao acesso aos programas terapêuticos com agonistas opiáceos. O presente artigo descreve os principais resultados para Portugal.

Material e Métodos: Os médicos que tratam doentes dependentes de opióides com recurso a programas terapêuticos com agonistas opiáceos foram inquiridos por entrevista directa; os doentes em programas terapêuticos com agonistas opiáceos, os doentes que não se encontravam em programas terapêuticos com agonistas opiáceos (em tratamento não farmacológico), e os utilizadores activos (pessoas dependentes de opióides que não se encontram actualmente em tratamento) receberam questionários de autopreenchimento.

Resultados: Verificou-se que, tanto os médicos como os doentes avaliam como 'Bom' o tratamento dos dependentes de opióides, em Portugal. O acesso ao tratamento foi considerado 'Fácil' pela maioria dos doentes e dos médicos, registando-se um elevado nível de satisfação entre os doentes. Contudo, o conhecimento dos doentes quanto às opções dos programas terapêuticos com agonistas opiáceos em Portugal encontra-se ainda abaixo do nível óptimo e as doses dos fármacos dos programas terapêuticos com agonistas opiáceos são frequentemente baixas.

Discussão: Os resultados do projecto ACCESS, no que diz respeito a PTAO, são encorajadores e evidenciam quer as áreas de sucesso, quer as áreas que podem ser melhoradas no futuro, no que diz respeito ao sistema de tratamento de dependências de opióides em Portugal.

Conclusões: O projecto ACCESS faz uma avaliação importante das atitudes e percepções em relação aos programas terapêuticos com agonistas opiáceos em Portugal. Estes dados deverão constituir um apoio importante a futuras tomadas de decisão quanto à optimização de respostas ao tratamento, no que diz respeito aos doentes e ao nível dos serviços de saúde.

Palavras-chave: Antagonistas de Narcóticos; Atitude do Pessoal de Saúde; Conhecimentos, Atitudes e Prática em Saúde; Dependência de Opióides; Portugal.

\section{ABSTRACT}

Background: There is a paucity of pan-European research on the state of medication-assisted treatment (MAT) from the perspective of the physicians who provide it, the patients who receive it and the opioid users who remain outside of the treatment system. Project ACCESS, a Europe-wide survey involving 11 countries, explored physicians', patients' and users' attitudes and beliefs regarding the quality of and access to medication-assisted treatment. This report presents key findings from Portugal.

Material and Methods: Physicians who treat opioid-dependent patients with medication-assisted treatment were interviewed faceto-face; patients in medication-assisted treatment, patients not in medication-assisted treatment (in therapy without medication), and users (opioid-dependent persons not currently in therapy) received self-completion questionnaires.

Results: The survey found that treatment for opioid-dependent individuals in Portugal is currently judged to be good by patients and physicians. Access to treatment was reported to be easy by the majority of patients and physicians and there was a high level of satisfaction among patients. However, patient awareness of medication-assisted treatment options is currently sub-optimal in Portugal and doses of medication-assisted treatment were frequently low.

Discussion: The results of the ACCESS project, as far as medication-assisted treatment is concerned, are encouraging and highlight both the success areas and those that need to be improved in the future within the system of treatment for opioid-dependent individuals in Portugal.

Conclusions: Project ACCESS provides a valuable assessment of attitudes and beliefs regarding medication-assisted treatment in Portugal. These data should help inform future decision-making to optimize responses to treatment at the patient and public health level.

Keywords: Health Knowledge, Attitudes, Practice; Attitude of Health Personnel; Narcotic Antagonists; Opioid-Related Disorders; Portugal.

\section{INTRODUÇÃO}

O uso ilícito de opióides constitui um importante problema de saúde pública em todo o mundo. A prevalência de utilizadores de opiáceos na Europa Ocidental e Central foi

estimada em 0,4 - 0,5\% (i.e., entre 1 e 1,4 milhões de pessoas). ${ }^{1}$ Em 2007, o United Nations Office on Drugs and Cri$\mathrm{me}^{2}$ estimava que, em termos globais, existiam entre 15,1 
e 21,1 milhões de pessoas que tinham utilizado opiáceos, pelo menos uma vez, durante o ano anterior; adicionalmente, em 2009, o World Drug Report declarou que os opiáceos continuavam a ser 'a droga mais problemática do mundo em termos de tratamento'. ${ }^{3}$

A dependência e o abuso de opióides estão associados a riscos significativos, não só para o utilizador, mas também para a sociedade em geral (e.g. risco de overdose, transmissão de doenças infecciosas, mortalidade prematura, furto e crime relacionado com a sua aquisição); o peso económico é igualmente elevado (e.g. perda de produtividade, custas judiciais e com a prisão, custos inerentes à saúde e ao bem-estar social). ${ }^{1,4-7}$ Apesar de o problema subjacente ser, por inerência, o mesmo, um conjunto de factores, incluindo pressões políticas, resultam em variações consideráveis nas estratégias adoptadas pelos diferentes países para combater os danos decorrentes da dependência de opióides $^{8,9}$ e do abuso de drogas em geral. ${ }^{10}$

A dependência de opióides foi, em tempos, considerada um problema exclusivamente social e comportamental, cuja resolução competia ao sistema de justiça criminal. Importantes avanços na compreensão da dependência de drogas e das estratégias para a sua prevenção e tratamento conduziram a um aumento da aceitação da dependência de opióides como um distúrbio mental crónico e recidivante, no qual os programas terapêuticos com agonistas opiáceos (PTAO) desempenham um papel importante..$^{1-3,8,10}$ É hoje amplamente aceite que os cuidados de longa-duração, que incluem intervenções farmacológicas e psicossociais, conduzem aos melhores resultados. ${ }^{2,3,8,11} \mathrm{Na}$ Europa, esta tendência é demonstrada, por exemplo, por uma maior disponibilidade de medidas para redução de riscos e minimização de danos (RRMD), tais como a introdução ou alargamento do acesso a PTAO e de programas de troca de seringas e agulhas. ${ }^{10}$ Todavia, os países diferem no modo e grau de implementação deste tipo de intervenções. ${ }^{10,12}$

Os diferentes países enfrentam um desafio comum, que reside na necessidade de se atingir um equilíbrio entre a maximização do acesso a um tratamento de elevada qualidade, garantindo, simultaneamente, a implementação de estratégias adequadas e seguras, de modo a manter um perfil de risco/benefício positivo aquando da prescrição de fármacos opióides. ${ }^{12} \mathrm{~A}$ abordagem terapêutica varia de país para país, nomeadamente no que diz respeito ao acesso ao tratamento (por via dos clínicos gerais/médicos de família [e.g. França] ou, primariamente, através de médicos especialistas [e.g. Itália]); se a toma de fármacos opióides é altamente supervisionada ou se os doentes têm a possibilidade de toma domiciliária; se estão ou não disponíveis programas de baixo limiar de exigência, no âmbito das medidas de RRMD; e na extensão de utilização das principais opções farmacológicas (metadona, buprenorfina e buprenorfina-naloxona). ${ }^{8}$ Estas diferenças podem ser influenciadas pela existência (ou não) de recomendações ou normas de orientação clínica nacionais e respectivo conteúdo, bem como pelos diferentes requisitos de formação dos médicos (e.g. na Alemanha, os médicos que pretendem tratar utiliza- dores de opióides têm de frequentar um curso de formação de 56 horas, ao passo que, em França, não é necessário qualquer tipo de formação adicional para médicos que pretendam prescrever buprenorfina). ${ }^{8}$

As variações nas políticas e práticas de tratamento entre os diferentes países podem também reflectir uma série de considerações locais, incluindo o contexto histórico, as prioridades de saúde pública, legislação e políticas nacionais, bem como a epidemiologia do consumo de drogas. Em Portugal existem diversos factores contextuais importantes que moldaram - e continuam a moldar - a direcção das políticas e práticas. Tal como outros países do Sul da Europa, Portugal atravessa, no presente, uma difícil situação económica (Nações Unidas, 2011), o que coloca pressão no orçamento da saúde. A avaliação do custo dos PTAO, na perspectiva dos utilizadores activos, dos doentes e dos médicos que os tratam, é crucial para a compreensão do seu valor e da necessidade de um investimento continuado que garanta que o tratamento se mantém acessível. Em Julho de 2001, entrou em vigor em Portugal uma lei que descriminalizou o consumo e posse para consumo de todas as drogas, incluindo a cocaína e a heroína. Isto significa que, apesar de o consumo destas drogas continuar a ser ilegal, este é tratado como uma infracção administrativa e não criminal. ${ }^{10}$ Um relatório do Cato Institute, de Washington, uma organização de investigação sobre políticas públicas, concluiu que a lei portuguesa para a descriminalização das drogas é a estratégia mais eficaz para reduzir a toxicodependência e os danos associados. ${ }^{10}$ Consequentemente, a redução da procura através da prevenção, da redução de riscos, da reinserção, e da maximização da disponibilidade de tratamentos e recursos surgem como pontos-chave do Plano Nacional Contra a Droga e a Toxicodependência para 2005/2012. ${ }^{10}$ O Instituto da Droga e da Toxicodependência (IDT), agora extinto, encontrava-se sob a tutela do Ministério da Saúde e era o organismo responsável pela elaboração e implementação das estratégias e planos de acção nacionais.

Estes factores tornam, assim, Portugal num caso de estudo extremamente interessante no contexto Europeu.

O facto de existirem várias opções de tratamento ${ }^{9}$ proporciona uma oportunidade de comparação entre diferentes sistemas de tratamento de dependência de opióides. No entanto, são escassos os estudos de investigação pan-europeus que reflectem o estado do tratamento na perspetiva do médico que o prescreve, do doente que o recebe e dos utilizadores activos de opióides que continuam fora do sistema terapêutico (quer voluntariamente, quer devido a outros factores, nomeadamente por falta de acesso adequado, ou por se encontrarem no contexto de um processo cíclico, repetido ao longo de vários anos, caracterizado por períodos com e sem tratamento, inclusão ou não em programas de minimização de danos, ou dentro e fora da prisão).

O projecto ACCESS é um estudo a nível europeu que envolveu 11 países com o objectivo de explorar as atitudes e percepções de médicos, doentes e utilizadores activos 
no que diz respeito à qualidade e ao acesso ao PTAO. Os resultados deste estudo pretendem apoiar a tomada de decisão informada relativamente às políticas e ao tratamento da dependência na Europa e, em última análise, sustentar o acesso dos doentes a cuidados de elevada qualidade. Os resultados globais para a Europa serão apresentados noutra publicação. Este artigo apresenta os principais resultados para Portugal, um país que tem sido observado de perto desde que adoptou a política de descriminalização, há uma década atrás, por outras partes do mundo que também procuram responder aos desafios colocados pela toxicodependência e suas consequências negativas para a sociedade e para a saúde pública.

Os dados deste inquérito permitem uma visão sobre o paradigma actual do tratamento e revelam em que sentido as políticas e as abordagens terapêuticas actuais poderão ser melhoradas. Especificamente, o estudo pretende avaliar se o sistema actual é bem-sucedido em assegurar que os doentes estão bem informados, têm acesso ao tratamento e recebem cuidados de qualidade; existe um equilíbrio ajustado entre os benefícios e os riscos do tratamento; o impacto da dependência de opióides na saúde pública é minimizado; e que o tratamento está disponível aos indivíduos reclusos em prisões.

\section{MATERIAL E MÉTODOS}

Todos os participantes deram o seu consentimento informado. Foram incluídos médicos que tratam doentes dependentes de opióides com PTAO, tendo sido inquiridos através de entrevista directa por técnicos da equipa do estudo em nome da empresa de estudos de mercado responsável pela recolha dos dados (Synovate Healthcare). O tempo médio por entrevista foi estimado em cerca de 25 minutos e as entrevistas foram realizadas entre 5 de Abril e 12 de Maio de 2011. Foram incluídos dois grupos de doentes: 1) indivíduos dependentes de opióides actualmente inseridos em PTAO; e 2) indivíduos dependentes de opióides actualmente a fazer tratamento sem PTAO. Foram ainda inquiridos utilizadores activos, definidos como indivíduos dependentes de opióides que não estavam a fazer tratamento. Os doentes e utilizadores activos não foram pré-avaliados tendo recebido questionários de auto-preenchimento, entregues de forma aleatória e recolhidos entre 5 de Abril e 13 de Junho de 2011.

O IDT disponibilizou à Synovate Healthcare uma lista de médicos credenciados para a prescrição de PTAO de opióides e uma lista de Equipas de Tratamento da toxicodependência e Comunidades Terapêuticas em diferentes cidades portuguesas.

Os utilizadores activos que não estavam incluídos em PTAO foram recrutados através das equipas de rua, conduzidas por ONGs e apoiadas financeiramente pelo IDT. De forma a suscitar interesse e aumentar a taxa de participação, foi enviada uma carta de recomendação para os médicos antes do início do estudo. Os participantes foram compensados pelo tempo dispendido e pela sua disponibilidade de cooperação: aos médicos foi atribuído um vou- cher no valor de $40 €$; às Comunidades Terapêuticas foram atribuídos donativos; os doentes e os utilizadores activos receberam um voucher de mercearia.

As entrevistas com os médicos permitiram recolher dados demográficos gerais (e.g. idade, sexo), perfil do médico (e.g. especialidade, qualificações), razões para tratarem os indivíduos dependentes de opióides com PTAO, comportamento actual em termos de tratamento e desenvolvimento futuro assumido (incluindo preocupações sobre o mau uso e desvio da medicação), opinião sobre os PTAO na região, sobre as recomendações/normas de orientação clínica e regulamentação de drogas, variabilidade regional da qualidade dos cuidados prestados aos doentes e importância dos programas de tratamento na prisão e no período pós-prisão. O questionário para os doentes dependentes de opióides e utilizadores incluía secções sobre dados demográficos e de caracterização geral (e.g. idade, sexo, estado civil, grau académico mais elevado, ocupação actual, estado de saúde actual), utilização de drogas e historial de tratamento, opinião sobre os PTAO, mau uso, desvio e prisão.

A dimensão estimada da amostra foi a seguinte:

- Médicos, $n=60$;

- Doentes em PTAO, $n=100$;

- Doentes que não estavam em PTAO, $n=50$;

- Utilizadores, $n=50$.

Todos os dados foram recolhidos pela empresa de estudos de mercado.

Para caracterizar os padrões de atitudes/percepções, as respostas ao inquérito foram inicialmente resumidas de forma descritiva (frequência e percentagens de respostas). Sempre que aplicável, foram utilizados testes estatísticos de diferenças entre grupos, testes-t bilaterais para valores médios ou testes- $z$ para percentagens, com um nível de significância $\alpha=0,05$. Os dados foram analisados através do Statistical Package for Social Sciences (SPSS).

\section{RESULTADOS \\ Características da população inquirida}

Conforme planeado, foram realizadas entrevistas a 60 médicos. Para garantir que as opiniões obtidas eram relativas a doentes tratados com diferentes opções dos PTAO, os questionários foram distribuídos até que as seguintes quotas estivessem completas: metadona, $80 \%$; buprenorfina e buprenorfina-naloxona, $20 \%$. No total, participaram no estudo 160 doentes. Preencheram os questionários 50 doentes que não estavam em PTAO e 50 utilizadores activos. Foram incluídas percentagens similares de médicos de ambos os sexos. A idade média foi de 51 anos e, em média, observaram 700 doentes por trimestre/ 11 doentes por dia. Dois terços $(66 \%)$ dos médicos entrevistados eram psiquiatras e cerca de um terço (32\%) eram médicos de clínica geral. No que respeita ao contexto, $82 \%$ dos médicos trabalhavam em Equipas de Tratamento do IDT. Em média, os entrevistados estavam credenciados há 14 anos, com 15 anos de prática clínica.

Nos três grupos de dependentes de opióides havia mais 
homens do que mulheres, especialmente entre os utilizadores activos. A idade média foi semelhante em todos os grupos. Apesar de muitas semelhanças, houve diferenças significativas entre os grupos: os doentes que não estavam em PTAO tinham um nível de educação superior $\left(12^{\circ}\right.$ ano ou superior) aos que estavam inseridos em PTAO; comparando com os outros grupos, havia mais utilizadores activos solteiros $(p<0,05)$ e desempregados $(p<0,05)$.

\section{Acesso ao tratamento}

A maioria dos doentes, quer no grupo de doentes em PTAO (94\%) quer no grupo dos que não estavam em PTAO $(82 \%)$, teve acesso ao tratamento através dos serviços do Estado (IDT) e a maioria dos doentes em PTAO (80\%) relataram que era 'muito fácil' ou 'fácil' encontrar um médico que lhes prescrevesse PTAO. Os médicos inquiridos estavam a tratar, em média, 155 doentes, sendo que $50 \%$ esperava que este número aumentasse nos próximos dois anos, essencialmente devido ao aumento previsto do número de indivíduos à procura de ajuda. A maioria $(88 \%)$ dos médicos considerou que é 'muito fácil' ou 'fácil' aceder a PTAO na sua área de actuação.

\section{Tratamento passado e actual}

Mais de metade dos doentes que não estavam actualmente em PTAO (56\%) e dos utilizadores activos (56\%) já tinham estado em PTAO anteriormente; o número médio de episódios de inclusão em PTAO anteriores para estes dois grupos foi de 2,0 e 2,7, respectivamente.

Nos doentes $(n=28)$ que não estavam a fazer PTAO mas que já o tinham feito anteriormente, o fármaco mais frequentemente utilizado no programa foi a metadona (46\%), seguido de buprenorfina (39\%) e $32 \%$ receberam outro tratamento. Relativamente aos utilizadores $(n=28)$ que tinham realizado PTAO anteriormente, o fármaco mais utilizado no programa foi a metadona (75\%); seguido de buprenorfina (18\%) e $39 \%$ tinham recebido outro tratamento.

Dos doentes em PTAO, 43\% relataram que tinham recebido tratamento anteriormente; $58 \%$ destes 69 indivíduos tinham recebido metadona, $46 \%$ buprenorfina e $30 \%$ receberam outro tratamento.

Considerando os indivíduos para quem o PTAO foi alterado ou interrompido, as consequências relatadas foram semelhantes nos três grupos (doentes em PTAO: $n=43$, doentes que não estavam em PTAO: $n=23$, utilizadores: $n=22$ ). Os eventos mais frequentemente reportados pelos doentes em PTAO, doentes que não estavam em PTAO e utilizadores activos foram a ânsia de consumir (craving), os aspectos sociais, a perda de rendimentos e os efeitos na saúde.

A Fig. 1 mostra a duração do tratamento actual. Na maioria dos doentes, o PTAO actual foi iniciado entre 1 e 5 anos atrás ( $51 \%$ dos doentes), tendo uma duração média de 5,7 anos.

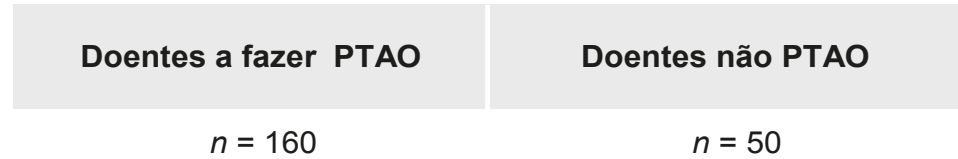

$$
n=160 \quad n=50
$$

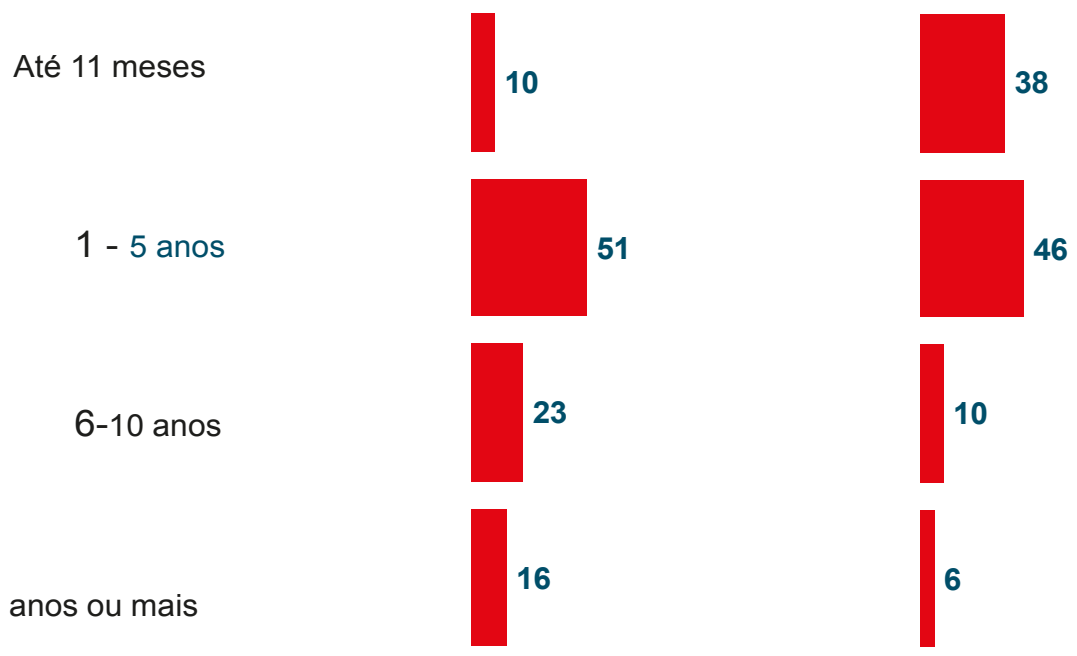
Média
5,7
3,9

Resultados em \%

Figura 1 - Duração do PTAO actual. Em média, os doentes com PTAO permanecem em tratamento durante 5,7 anos, enquanto doentes sem PTAO permanecem em tratamento durante menos tempo. 


\section{Motivações e objectivos do tratamento}

As razões mais frequentemente apontadas pelos doentes para iniciar o PTAO foram a vontade de terminar de vez com a sua dependência e o desejo de melhorar a sua saúde. Outras razões igualmente referidas foram a vontade de cuidar melhor das suas famílias e de fazer uma mudança nos círculos sociais onde se inserem. As razões para iniciar o PTAO foram semelhantes nos doentes em PTAO e nos doentes que não estavam em PTAO (Fig. 2). Os médicos consideram que os objectivos mais importantes dos PTAO são reduzir os riscos para a saúde, reduzir as co-morbilidades e a estabilização social do doente (Fig. 3).

\section{Factores que determinaram a escolha da medicação}

Os factores clínicos foram definidos pelos médicos como os mais relevantes na escolha da medicação, nomeadamente a adequação às co-morbilidades do doente, eficácia, segurança/ tolerabilidade e risco de interacções medicamentosas (Fig. 4).

A metadona foi a medicação mais utilizada (prescrita em $65 \%$ dos casos), mas tal foi maioritariamente impulsionado pelos custos associados e não pelos factores clínicos. Os médicos referem que $69 \%$ dos seus doentes pedem um determinado fármaco, tendo acedido ao pedido em $60 \%$ dos casos. No estudo, os dados obtidos através dos doentes relativamente à escolha da medicação foram discordantes face aos fornecidos pelos médicos: $30 \%$ dos doentes em PTAO afirmaram ter pedido um determinado fármaco, tendo esse pedido sido atendido em $77 \%$ dos casos. Não foi dada a possibilidade de escolha do medicamento a um terço dos doentes. Quando questionados acerca do seu conhecimento sobre as opções de tratamento antes de o iniciarem, cerca de um terço (32\%) dos doentes em PTAO referiram falta de conhecimentos, auto classificando-se como 'muito pouco informados' ou 'não muito informados'. Em relação ao conhecimento prévio dos diferentes fármacos, $89 \%$ dos doentes em PTAO tinham conhecimento da metadona líquida antes de iniciarem o tratamento. Uma proporção elevada de indivíduos conhecia a buprenorfina em monoterapia (79\%), mas menos de metade (44\%) conhecia a combinação buprenorfina-naloxona (Fig. 5).

A maioria dos doentes procurou informações sobre as opções de tratamento antes de iniciar o programa (74\% dos doentes em PTAO e $80 \%$ dos doentes que não estavam em PTAO). A equipa de tratamento foi a principal fonte de informação sobre as opções de tratamento para doentes em PTAO (59\%), para doentes que não estavam em PTAO (73\%) e utilizadores activos (84\%). Outros utilizadores activos de drogas constituíram igualmente uma importante fonte de informação (para $45 \%$ dos doentes em PTAO, 43\% dos doentes que não estavam em PTAO e $62 \%$ dos utilizadores activos). Estes resultados revelam que o 'passa palavra' é uma forma relevante de divulgar o tema.

\section{Dosagem}

Houve uma grande variação nas doses diárias entre os vários doentes estudados, mas verificou-se uma tendência geral para doses baixas. Por exemplo, $60 \%$ dos doentes tratados com buprenorfina ou buprenorfina-naloxona estavam a tomar uma dose média $\leq 4 \mathrm{mg}$ e nenhum tinha tomado uma dose superior a $8 \mathrm{mg}$. Mais de um terço dos doentes (36\%) tratados actualmente com metadona estava a tomar uma dose $\leq 32,5 \mathrm{mg}$.
Queria acabar de vez com a minha dependência

Queria melhorar a minha saúde

Queria cuidar da minha família

Financiar o consumo de droga é demasiado caro

Queria fazer uma mudança nos círculos sociais onde me insiro

Estava preocupado em contrair uma infecção/ doença

Queria reduzir o consumo porque estava a consumir demais

Precisava de uma pausa no vício porque as coisas estavam caóticas

Queria (ser capaz) de trabalhar outra vez

Queria para de cometer crimes por causa da minha dependência

Tinha medo de apanhar uma overdose

Tinha medo de perder o emprego

Estava preocupado com alguma acusação/ prisão

Gravidez

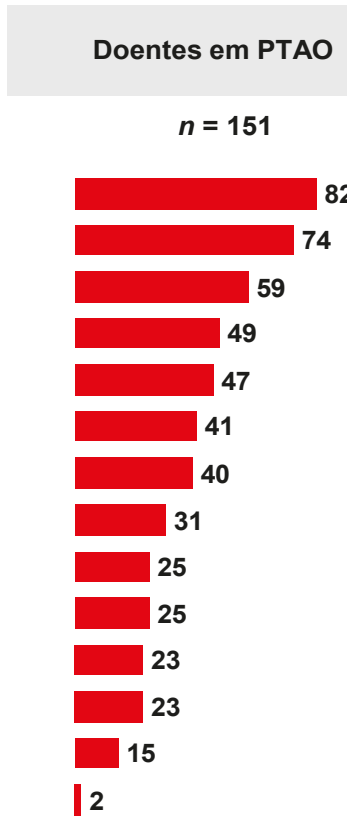

82

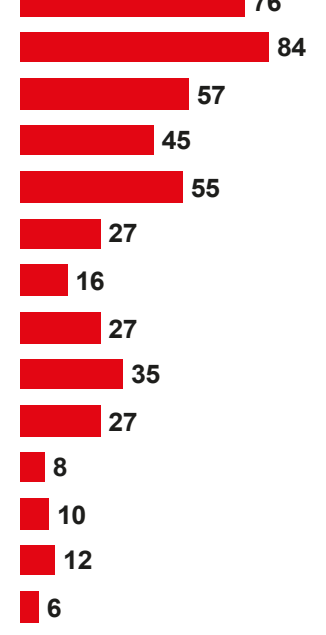

Resultados em \%

Figura 2 - Razões para iniciar o PTAO. Os doentes não PTAO apontaram como principal motivação para iniciarem o tratamento o desejo de melhorarem a sua saúde; a mesma motivação foi muito importante para os doentes em PTAO, apesar destes terem referido em primeiro lugar o desejo de acabar de vez com a dependência. 


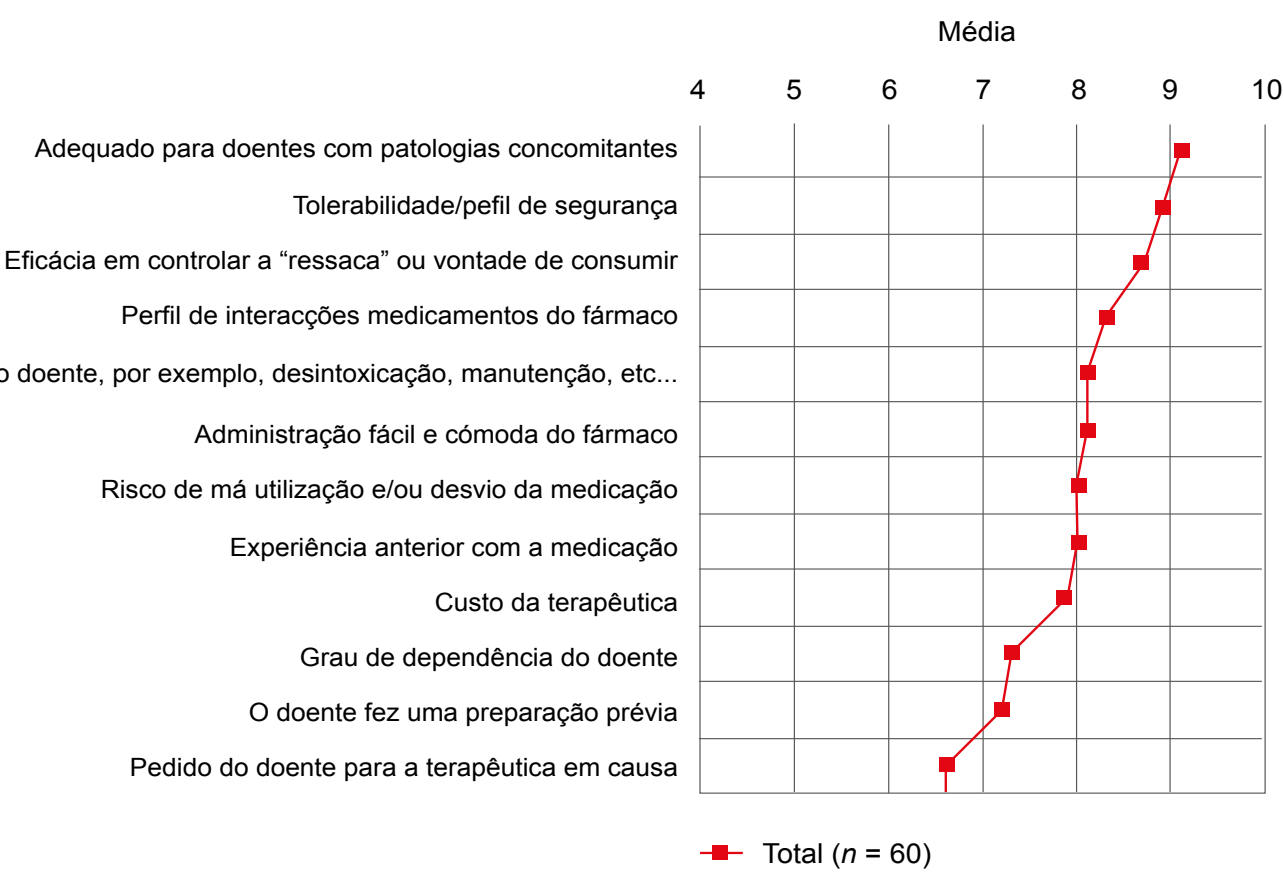

Figura 3 - Objectivos da terapia. Os médicos consideram que os objectivos mais importantes dos PTAO são reduzir os riscos para a saúde, reduzir as co-morbilidades e a estabilização social do doente. A terapiêutica de manutenção a longo prazo aparece como o objectivo menos importante.

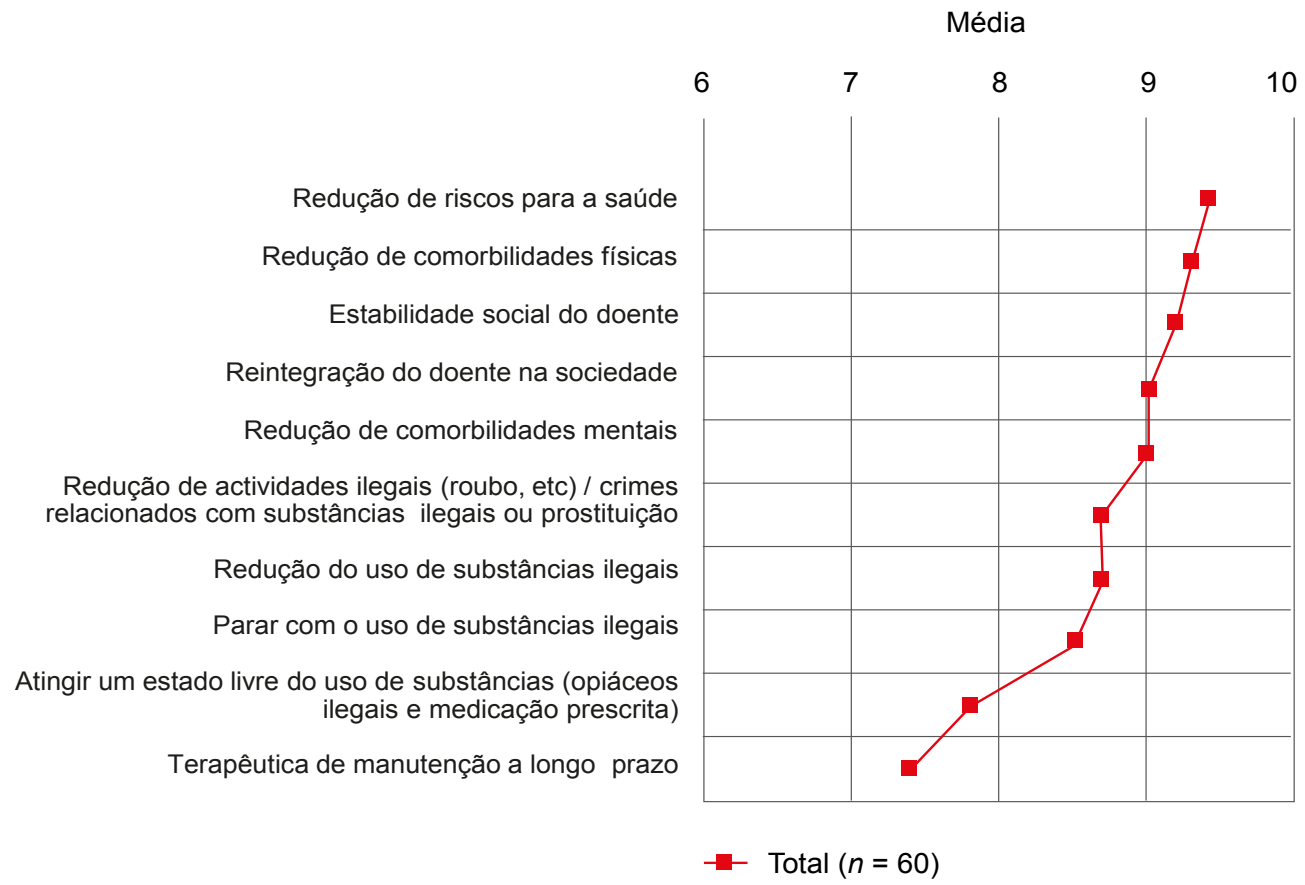

Figura 4 - Factores determinantes na escolha da medicação. Os factores clínicos definidos pelos médicos como os mais relevantes na escolha da medicação foram a sua adequação às co-morbilidades do doente e a eficácia, segurança/ tolerabilidade e risco de interacções medicamentosas.

\section{Tomas Supervisionadas}

Pelo menos a um nível parcial, $40 \%$ dos doentes receberam o seu tratamento de forma supervisionada. Mais de um quarto dos doentes em PTAO (28\%) referiram que todas as tomas estavam sob supervisão do médico ou do farmacêutico, enquanto a $12 \%$ dos doentes era-lhes permitido fazer toma domiciliária aos fins-de-semana e/ou nas férias. $O$ relato dos médicos relativamente à supervisão de doses é concordante com o dos doentes, sendo que os médicos referiram que $36 \%$ dos seus doentes são supervisionados diariamente, $22 \%$ fazem a medicação em casa aos fins-de-semana ou feriados e os restantes fazem a medicação em casa frequentemente.

O requisito para a supervisão das tomas foi referido por $19 \%$ dos doentes em PTAO como sendo a regra para início de tratamento mais difícil de cumprir. Comparativamente, 
$25 \%$ e $24 \%$ dos doentes, respectivamente, referiram que ter de parar completamente o consumo de drogas e estar livre de drogas a longo prazo foram as regras mais difíceis de cumprir. Cerca de um terço $(30 \%)$ referiu que ser obrigado a apresentar-se em todas as marcações foi a regra de continuação do tratamento com maior impacto no seu dia-a-dia. Cerca de $21 \%$ dos doentes referiram que a necessidade de estarem livres de drogas a longo prazo (21\%) e o facto de terem de parar completamente o consumo de drogas $(21 \%)$ constituíram as regras de continuação do tratamento com maior impacto na sua vida diária.

\section{Apoio psicossocial}

A percentagem de doentes que não estava em PTAO e que recebia apoio psicossocial foi de $86 \% ; 42 \%$ dos doentes que estavam em PTAO não estavam a receber apoio psicossocial. A maioria dos doentes que estava a receber aconselhamento psicossocial considerou-o 'muito útil' ou 'útil' (48\% e $45 \%$ dos doentes em PTAO e $77 \%$ e $23 \%$ dos doentes que não estavam em PTAO, respectivamente). $\mathrm{O}$ apoio psicossocial foi largamente considerado útil para manter a motivação para continuar no programa de tratamento $(74 \%$ dos doentes em PTAO e $88 \%$ dos doentes que não estavam em PTAO). Uma percentagem elevada de doentes referiu que não teria continuado no programa se não tivesse tido o apoio psicológico e social $(28 \%$ dos doentes em PTAO e $42 \%$ dos doentes que não estavam em PTAO). O aconselhamento psicossocial foi também considerado útil no que diz respeito a aspectos práticos, nomeadamente para encontrar emprego ou casa $(26 \%$ dos doentes em PTAO e $26 \%$ dos doentes que não estavam em PTAO). Poucos doentes referiram que o aconselhamento não os ajudou em nada ( $6 \%$ dos doentes em PTAO e $3 \%$ dos doentes que não estavam em PTAO).

\section{Satisfação com os programas de tratamento}

A maioria dos doentes estava satisfeita com o sucesso do seu tratamento: $50 \%$ dos doentes em PTAO estavam satisfeitos e $39 \%$ estavam muito satisfeitos. Dos doentes que não estavam em PTAO, $40 \%$ estavam satisfeitos e $54 \%$ estavam muito satisfeitos. Dos doentes em PTAO, a taxa de satisfação mais elevada estava associada ao tratamento com buprenorfina-naloxona (94\% estavam muito satisfeitos ou satisfeitos com o seu PTAO comparando com $89 \%$ dos doentes tratados com buprenorfina e com $85 \%$ dos doentes tratados com metadona). A maioria dos médi$\cos (51 \%)$ considera que os doentes estão satisfeitos com o tratamento prescrito na sua zona. Grande parte dos médicos $(65 \%)$ estava satisfeita com os programas de tratamento na sua zona; a burocracia reduzida (sem listas de espera e boa organização) foi referida como a principal razão para a sua satisfação (68\%); a correspondência entre a oferta e a procura foi outra razão apontada como importante (34\%).

\section{Mau uso/ Desvio}

Quase todos os médicos (92\%) estavam 'muito preocupados' (45\%) ou 'algo preocupados' (47\%) com o mau uso e o desvio, sendo que $78 \%$ afirmaram ter tentado encontrar uma solução para o problema. (e.g. alterar a dose) quando se apercebiam que um doente estava a utilizar incorrectamente ou a desviar a sua medicação. O desvio e o mau uso da medicação foram reportados por $14 \%$ e $10 \%$ dos doentes em PTAO, respectivamente, sendo que $16 \%$ dos utilizadores activos confirmaram comprar medicação desviada. O mau uso dos fármacos dos PTAO foi maioritariamente reportado pelos doentes tratados com metadona com, respectivamente, $100 \%$ e $97 \%$ dos doentes dos grupos da buprenorfina e buprenorfina-naloxona a afirmarem que nunca tinham utilizado mal a sua medicação. Adicionalmente, enquanto $11 \%$ dos doentes do grupo da metadona revelaram

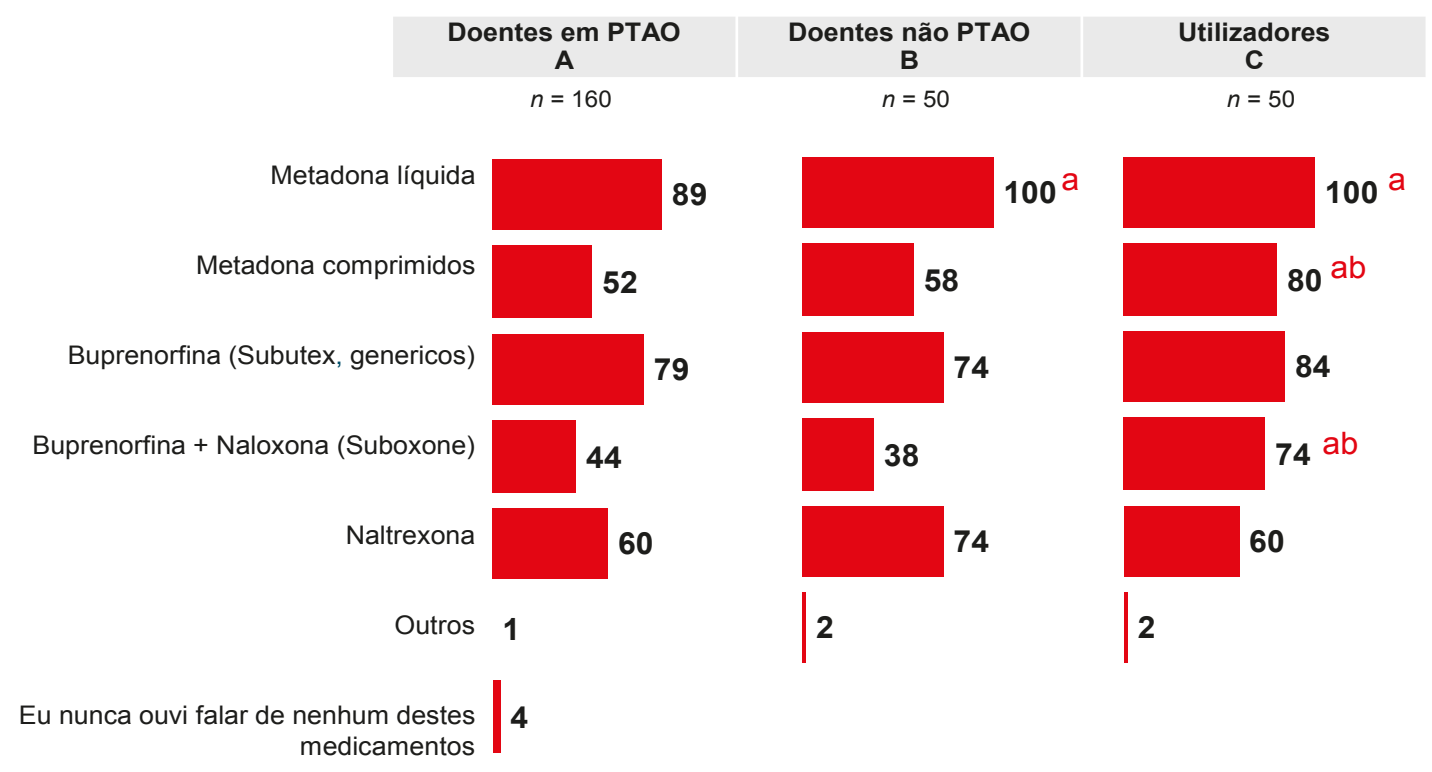

Figura 5 - Conhecimento prévio dos diferentes fármacos. Os doentes em PTAO revelaram maior conhecimento prévio dos diferentes fármacos, enquanto os doentes não PTAo referiram falta de conhecimento. O Suboxone é o fármaco menos conhecido. 


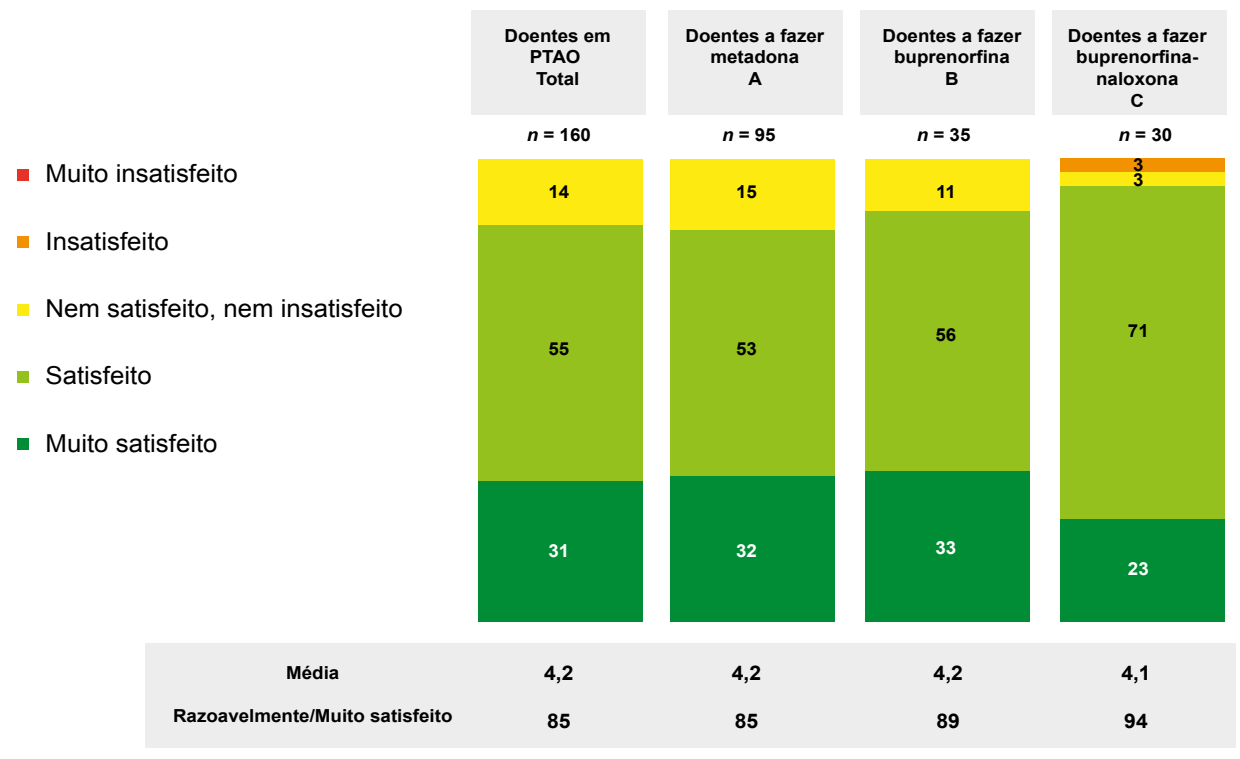

Figura 6 - Satisfação com os Programas de Tratamento.

que injectavam a sua medicação, ninguém nos dois grupos da buprenorfina o reportou. Os doentes em PTAO afirmaram que a metadona líquida era o fármaco dos PTAO mais fácil de encontrar nas ruas, com $72 \%$ dos doentes a reportarem que a metadona líquida era 'muito fácil' ou 'fácil' de encontrar. De igual modo, de entre os fármacos usados nos PTAO, a buprenorfina-naloxona era o menos acessível, com $39 \%$ dos doentes a afirmarem que era 'muito fácil' ou 'fácil' encontrá-lo.

\section{Consumo paralelo de drogas}

Quase um terço dos doentes em PTAO (32\%) reportou o consumo paralelo de drogas. No grupo de doentes tratados com buprenorfina-naloxona verificou-se uma incidência significativamente mais baixa de consumo paralelo de drogas $(16 \%)$ versus os que eram tratados com metadona $(36 \% ; p<0,05)$. As razões mais frequentemente apresentadas para o consumo paralelo de drogas eram a necessidade de ocasionalmente 'ficarem pedrados' (referido por $60 \%$ dos doentes que reportaram o consumo paralelo de drogas), o facto de o PTAO não controlar a necessidade de consumir (referido por $20 \%$ dos doentes que reportaram o consumo paralelo de drogas), ou a necessidade de consumir quando falhavam uma consulta (referido por $16 \%$ dos doentes que reportaram o consumo paralelo de drogas) (Fig. 6).

\section{O PTAO nas prisões}

Vinte por cento dos doentes e utilizadores já tinham estado presos, estando a maioria dos motivos (85\%) relacionado com drogas (incluindo cometer crimes para sustentar os consumos). Metade dos doentes que estiveram presos não recebeu tratamento enquanto esteve preso $\mathrm{e}$ cerca de metade dos doentes que foram presos enquanto estavam em PTAO (48\%) mudaram de medicação ou pa- raram completamente o tratamento durante esse período. Todos os médicos inquiridos consideram que disponibilizar as mesmas opções de tratamento nas prisões é 'importante' ou 'muito importante'. A continuidade do tratamento foi também vista como relevante, sendo que todos os médicos consideram 'muito importante' ou 'importante' tratar estes doentes depois de saírem da prisão.

\section{Obstáculos ao acesso e qualidade do tratamento}

Um elevado número de médicos considerou que a baixa disponibilidade de médicos na sua área (55\%), o estigma (53\%), os custos associados ao tratamento (43\%) e as regras inflexíveis do mesmo (32\%) eram os obstáculos mais importantes para o tratamento na sua região. O factor mais frequentemente identificado pelos doentes em PTAO como potencial incentivo ao início mais precoce do tratamento em PTAO foi terem mais informação sobre as opções de tratamento (42\%) seguido por uma maior flexibilidade das regras $(22 \%)$.

\section{Importância das recomendações/ normas de orienta- ção clínica}

A maioria dos médicos considera as normas de orientação clínica como 'importantes' ou 'extremamente importantes'. Os mesmos médicos consideram que, a existirem, as recomendações seriam uma 'referência' ou uma 'referência muito importante' no seu trabalho. Dois terços dos médi$\cos (66 \%)$ reportaram que as recomendações iriam tornar o seu trabalho 'muito mais fácil' ou 'algo mais fácil'. Quando questionados sobre a variabilidade regional da qualidade dos cuidados dos doentes, muitos médicos (45\%) consideram que existem grandes diferenças inter-regionais, em Portugal. Os médicos consideram como prioridade fundamental na sua área, a melhoria da cobertura/condições de fornecimento $(71 \%)$ e prevenção $(21 \%)$. 


\section{DISCUSSÃO}

Os resultados do projecto ACCESS permitem fazer uma avaliação das atitudes e percepções de médicos, doentes e utilizadores activos Portugueses, no que diz respeito a PTAO. Os resultados do estudo são encorajadores e evidenciam, quer as áreas de sucesso, quer as áreas que podem ser melhoradas no futuro, no que diz respeito ao sistema de tratamento de dependências de opióides em Portugal. Estes resultados poderão ser utilizados de forma a auxiliar futuras tomadas de decisão no que diz respeito a optimizar a resposta ao tratamento da dependência de opióides.

$\mathrm{O}$ acesso a PTAO parece ser bom, sendo que a maioria dos doentes em PTAO (80\%) e dos médicos (88\%), reportaram que o acesso ao tratamento é fácil ou muito fácil para os doentes. Este resultado poderá sustentar outros resultados positivos obtidos no questionário, nomeadamente, de que o PTAO tem que ser generalizado para que os seus benefícios sejam sentidos pelos indivíduos e pela sociedade em geral. Outro resultado encorajador é que os doentes em PTAO reportaram um elevado grau de satisfação com a sua medicação (desde $85 \%$ a fazer metadona até $94 \%$ a fazer buprenorfina-naloxona). Oitenta e nove por cento dos doentes em PTAO reportaram igualmente que estavam satisfeitos com o sucesso do seu programa de tratamento. Dado que os doentes apresentaram diferentes razões para procurarem tratamento - melhorar a sua saúde, alterar os seus círculos sociais - o elevado grau de satisfação apresentado por estes é encorajador, uma vez que sugere que o tratamento está a ter um impacto positivo em diversas áreas da sua vida. $\mathrm{O}$ facto de que a grande maioria dos doentes diminuiu significativamente o consumo de drogas ilícitas demonstra, em termos práticos, os benefícios do actual tratamento: dois terços dos doentes em PTAO reportaram nunca ter utilizado drogas ilícitas, sendo que apenas uma minoria (5\%) afirmou consumir diariamente este tipo de drogas.

Os resultados positivos do questionário podem ser, em parte, explicados pela política de descriminalização do consumo de drogas existente em Portugal, que proporciona condições para que os doentes, mais facilmente, aceitem entrar num programa de tratamento. Um relatório do CATO Institute, sobre a descriminalização do consumo de drogas em Portugal, ${ }^{10}$ afirma que Portugal incentivou os dependentes de opióides a procurar tratamento, por já não recearem acções judiciais, ou serem presos devido à utilização de drogas ilícitas. No relatório foi também sublinhado que recursos anteriormente alocados a acções judiciais para com os dependentes de opióides ficaram agora disponíveis para o tratamento da dependência de drogas ilícitas. ${ }^{10}$ Os PTAO estão generalizados em Portugal, principalmente através dos serviços especializados públicos, mas também de alguns hospitais, sendo ainda possível aceder às doses em centros de saúde e farmácias. Em 2008, cerca de 26.000 indivíduos estavam registados no programa de PTAO, contrastando com os cerca de 23.000 indivíduos registados em 2006. ${ }^{13}$ Os serviços públicos são gratuitos em Portugal e acessíveis para todos os dependentes de opióides que procurem tratamento; ${ }^{13}$ no entanto, indivíduos a quem seja prescrita buprenorfina ou buprenorfina-naloxona necessitam de fazer um co-pagamento de $63 \%$ do preço do medicamento, o que representa cerca de $60 €$ por mês para uma dose média diária de $8 \mathrm{mg}$. O resultado do estudo realizado aponta para o facto de que médicos e doentes consideram que o acesso ao tratamento é bom e indicia que as políticas adoptadas em Portugal estão a funcionar em termos práticos.

No entanto, apesar da existência de evidências de que o sistema actual de tratamento está a ser bem-sucedido em várias áreas importantes, Portugal está, neste momento, a enfrentar uma situação económica difícil, sendo que parte das medidas de austeridade implementadas implicam uma grave redução da despesa com Saúde. Neste contexto, os resultados deste estudo fornecem evidências importantes de que, de acordo com médicos e doentes em tratamento, os PTAO estão a ter um impacto positivo nos doentes. Estes resultados sublinham a necessidade de continuar o investimento com PTAO e, ao mesmo tempo, identificam certas áreas passíveis de melhorias futuras, que permitam aumentar ainda mais os benefícios do tratamento. As actuais pressões económicas podem também resultar numa alteração no padrão de prescrição na direcção de opções associadas a economias de custo.

Para se tirar o máximo partido das alternativas medicamentosas disponíveis para PTAO, é imperativo que cada doente discuta com o seu médico as opções disponíveis para identificar a estratégia que mais se ajusta às suas necessidades individuais. Poderá ser atingido um maior empenho no tratamento se o doente for activamente envolvido e responsabilizado na definição do seu plano de tratamento. ${ }^{14}$ De facto, actualmente, é considerado essencial envolver activamente o doente no seu plano de tratamento, estando esta abordagem associada a melhores resultados. ${ }^{15}$ Este estudo revelou que alguns doentes estão a ser envolvidos na escolha do seu tratamento, sendo que existe uma elevada proporção de doentes que refere que a sua medicação foi seleccionada por si. No entanto, mais de um quinto dos doentes não tinha conhecimento de buprenorfina em monoterapia e mais de metade não tinha conhecimento de buprenorfina-naloxona. Um terço dos doentes reportou não ter sido envolvido no processo de escolha. Desta forma, estes resultados ilustram uma importante falta de conhecimento dos doentes em relação às alternativas terapêuticas disponíveis, situação que necessita de ser corrigida para melhorar os resultados do tratamento. Esta falha é particularmente preocupante dado que muitos doentes e utilizadores activos de drogas ilícitas já tinham tido anteriormente outros ciclos terapêuticos, o que sugere que podem estar a ter repetidamente acesso ao sistema de tratamento sem que nunca lhes tivessem sido apresentadas todas as alternativas terapêuticas. Por exemplo, $75 \%$ dos utilizadores activos reportaram que, na média, $2,7 \mathrm{ci}-$ clos de tratamentos prévios envolveram a utilização de metadona. Adicionalmente é provável que muitos indivíduos, 
de facto, não tenham hipótese de escolha porque estão limitados à metadona por razões puramente económicas, dado que não existe um co-pagamento associado a esta opção de PTAO.

Uma vez seleccionada a medicação e iniciado o tratamento, é crucial que os doentes sejam induzidos de forma segura e que sejam estabilizados numa dose efectiva de cada fármaco. Diversos autores, baseados em pesquisa bibliográfica, defendem que doses de metadona de, pelo menos, $80 \mathrm{mg} /$ dia têm uma maior probabilidade de serem óptimas em termos de se atingir, não só uma melhoria dos sintomas de privação/ vontade de consumir, mas também um nível suficiente de tolerância cruzada que diminui os efeitos e, deste modo, o consumo ilícito de opióides. A buprenorfina tem propriedades farmacológicas únicas que lhe conferem uma lenta dissociação dos receptores opióides mu e um tempo de acção longo. Doses elevadas de buprenorfina permitem atingir um bloqueio duradouro dos receptores mu contra a acção de outros opióides consumidos concomitantemente. ${ }^{16}$ Vários estudos demonstraram que existe uma relação causal entre a dose de buprenorfina, ou de buprenorfina-naloxona, e os resultados obtidos, sendo que doses mais elevadas estão associadas a uma maior taxa de retenção em tratamento, ${ }^{17,18}$ e uma maior redução do consumo ilícito de opióides é evidente para doses de $16 \mathrm{mg}$ comparativamente a doses de $8 \mathrm{mg} .{ }^{19}$ Outros estudos anteriores sugerem que $80 \%$ dos receptores $m u$ devem ser inactivados para poder haver o bloqueio dos efeitos da heroína, e que doses de $2 \mathrm{mg}, 16 \mathrm{mg}$ e de $32 \mathrm{mg}$ estão associadas a uma menor disponibilidade dos receptores $m u$ de, respectivamente, $41 \%$, $80 \%$ e $84 \% .{ }^{20}$ Contudo, estes efeitos variam ao longo do tempo, tendo sido sugerido noutro estudo que $16 \mathrm{mg}$ de buprenorfina estão associados a níveis de disponibilidade dos receptores mu de 30\% após quatro horas e $54 \%$ após 28 horas. ${ }^{17}$

De acordo com a literatura, é sugerido que alguns dos doentes incluídos neste estudo podem estar a tomar doses inferiores às terapêuticas, sendo que $60 \%$ dos doentes a receber buprenorfina ou buprenorfina-naloxona, estavam a tomar doses $\leq 4 \mathrm{mg}, 64 \%$ dos doentes a receber metadona estavam a tomar doses $\leq 75 \mathrm{mg}$ e mais de um terço dos doentes estavam a tomar doses $\leq 32,5 \mathrm{mg}$. Este achado está de acordo com os resultados de um estudo efectuado recentemente, que sugere que são tendencialmente prescritas doses baixas de metadona e buprenorfina na Europa. $8,21 \mathrm{Um}$ estudo recente sobre as práticas de tratamento, realizado em Itália, Alemanha, França e no Reino Unido, revelou que estes resultados se desviam do identificado pela medicina baseada na evidência, em que as doses de indução e manutenção sub-terapêuticas são comuns. ${ }^{8}$ Em Portugal as normas de orientação clínica para PTAO estão actualmente a ser discutidas. De acordo com os médicos que responderam a este questionário, a existência de normas de orientação clínica é muito importante pois, caso existissem, facilitariam o seu trabalho. Deste modo, o desenvolvimento de normas de orientação clínica baseadas na evidência pode ser uma oportunidade para melhorar ainda mais a qualidade dos serviços já existentes em Portugal.

Uma vez atingida a dose de estabilidade, é importante manter os doentes em tratamento e minimizar o consumo de drogas ilícitas durante um período de tempo tal que thes dê oportunidade de realizar os objectivos a longo prazo do tratamento, incluindo melhoria da sua saúde e das suas competências sociais. Dois factores-chave que têm impacto no acima descrito incluem a disponibilização de aconseIhamento psicossocial, de modo a aumentar os benefícios da medicação, e a forma como os doentes encaram os requisitos do tratamento (e.g. teste de urina, toma supervisionada) como tendo um impacto aceitável na sua vida. $O$ presente estudo revela que muitos dos doentes em PTAO não têm aconselhamento psicossocial (42\%) e que aqueles que têm este tipo de aconselhamento consideram-no muito útil, não só como forma de motivação para continuar a fazer o tratamento, mas também como ajuda em aspectos práticos no âmbito da reinserção, como por exemplo na procura de emprego ou habitação. Deste modo, estes resultados confirmam os benefícios do aconselhamento e apoio psicossocial e evidenciam que os doentes devem ser encorajados a recorrer ao mesmo como parte integrante do PTAO.

De acordo com os resultados deste estudo, a toma supervisionada foi considerada a regra mais difícil de cumprir no início da terapêutica por uma grande proporção de doentes. Uma vez iniciado o tratamento, ter que estar presente em todas as marcações, foi a regra apontada por um maior número de doentes como tendo um maior impacto na sua vida quotidiana. Mais ainda, uma maior flexibilidade nas regras foi referida pelos doentes como sendo o segundo factor de encorajamento mais relevante para um início mais precoce do tratamento. Apesar de os doentes estarem a fazer o tratamento, em média, há 5,7 anos, cerca de um terço $(28 \%)$ tinha a toma supervisionada e $12 \%$ recebiam doses para tomar em casa apenas aos fins-de-semana e durante as férias. Apesar de a toma ser supervisionada numa proporção elevada de doentes, $92 \%$ dos médicos mostraram preocupação relativamente ao mau uso e desvio de medicação. No geral, $10 \%$ dos doentes afirmaram ter feito mau uso da medicação e $14 \%$ dos mesmos afirmaram tê-la desviado. O mau uso da medicação foi predominante referido por doentes a tomar metadona, sendo que, no que diz respeito ao desvio, a metadona líquida foi referenciada pelos doentes como a medicação que mais facilmente se encontra na rua. Globalmente, os resultados deste estudo sugerem que os doentes consideram difícil cumprir os requisitos de assiduidade e toma supervisionada mas que o mau uso e desvio ocorrem, apesar dos níveis actuais de supervisão. Considerando o custo crescente associado à toma diária supervisionada, sempre que possível, a toma domiciliária pode constituir uma estratégia mais custo-efectiva, com benefícios na aceitação dos doentes. Um aumento de PTAO mais adequado a tomas domiciliárias (e.g. buprenorfina, buprenorfina-naloxona) ou a tomas não supervisionadas poderá levar a uma redução de custos. ${ }^{22-24}$ 
Por exemplo, num estudo feito por Bell et $\mathrm{al}^{22}$ comparou-se a relação custo-efectividade dos regimes de buprenorfina-naloxona, com e sem toma supervisionada no contexto da dependência de heroína. Este estudo demonstrou que os tratamentos com um seguimento clínico próximo, mas sem toma supervisionada, eram significativamente mais económicos do que os tratamentos com toma supervisionada. Apesar de, neste estudo, poucos doentes terem referido fazer mau uso da medicação prescrita ou desviá-la, a adopção de PTAO que estejam associados a menor mau uso e desvio de medicação deve ser considerada como uma forma de ajuda para a redução destes problemas. Aproximadamente dois terços dos médicos em Itália, Alemanha e no Reino Unido concordaram ser essencial a prescrição de medicação com o menor potencial de desvio. ${ }^{8} \mathrm{O}$ aumento esperado da necessidade de PTAO, em combinação com a presente situação económica portuguesa, sugere que as opções de tratamento mais custo-efectivas serão mais relevantes.

Um dos objectivos primários dos PTAO é o de reduzir o consumo de drogas ilícitas. Os resultados deste estudo confirmam que a maioria dos doentes obtiveram benefícios significativos com o tratamento, sendo que $68 \%$ destes afirmaram 'nunca' ter recorrido ao consumo adicional de drogas. De entre o quase $1 / 3$ dos doentes que, no presente estudo, reportaram consumo paralelo de drogas, razões frequentemente identificadas para este comportamento foram sentir vontade de 'ficarem pedrados' ou quando falhavam uma marcação. É possível que o consumo paralelo reportado neste estudo possa ser, em parte, uma consequência de nos PTAO se terem utilizado doses insuficientes para impedir a ânsia de consumir (craving). É de salientar que os doentes tratados com buprenorfina-naloxona referiram um consumo paralelo de drogas significativamente menor aos doentes tratados com metadona. A menor incidência de consumo paralelo de drogas deverá ser explorada de forma a determinar se os doentes tratados com buprenorfina-naloxona têm menos comportamentos compulsivos ou de menor intensidade, do que os doentes tratados com metadona.

\section{REFERÊNCIAS}

1. Maremmani I, Pacini M, Pani PP, on behalf of the 'Basics on Addiction Group'. Basics on addiction: a training package for medical practitioners or psychiatrists who treat opioid dependence. Heroin Addict Relat Clin Probl. 2011;13:5-40.

2. United Nations Office on Drug and Crime and World Health Organization (2008). Principles of drug dependence treatment. [Consultado 2011 Nov 14]. Disponivel em http://www.unodc.org/documents/drug-treatment/ UNODC-WHO-Principles-of-Drug-Dependence-Treatment-March08. pdf.

3. United Nations Office on Drugs and Crime (2009). World Drug Report. [Consultado 2011 Nov 14]. Disponível em http://www.unodc.org/documents/wdr/WDR_2009/WDR2009_eng_web.pdf.

4. Yokell MA, Zaller ND, Green TC, Rich JD. Buprenorphine and buprenorphine/naloxone diversion, misuse, and illicit use: an international review. Curr Drug Abuse Rev. 2011;4:28-41.

5. Stotts AL, Dodrill CL, Kosten TR. Opioid dependence treatment: options in pharmacotherapy. Expert Opin Pharmacother. 2009;10:1727-40.

6. United Nations (2011). World economic situation and prospects 2011
O acesso ao tratamento nas prisões é cada vez mais reconhecido como uma oportunidade importante mas frequentemente perdida para abordar o problema do consumo ilícito de opióides e reduzir os custos associados à passagem dos doentes pelo sistema judicial. A falta de continuidade do tratamento reportada pelos indivíduos encarcerados e a importância a ela atribuída pelos médicos indica que a disponibilidade de PTAO nas prisões portuguesas deve ser melhorada. Adicionalmente, o alargamento do formulário das prisões para incluir outras opções de tratamento para além da metadona deve ser considerado. Um estudo sobre o consumo de drogas e PTAO nas prisões concluiu que os doentes com elevado risco de danos associados à utilização de drogas merecem uma abordagem terapêutica e atitudes que deverão ser, pelo menos, iguais às disponibilizadas a consumidores inseridos na comunidade. ${ }^{25}$

\section{CONCLUSÕES}

Inevitavelmente, a metodologia usada está associada a potenciais vieses em termos de conseguir uma amostra representativa (viés de selecção) e a confiança de dados auto-relatados. No entanto, os resultados obtidos através das respostas dos médicos, doentes e utilizadores, fornecem importantes elementos para a avaliação das atitudes e percepções no que diz respeito a PTAO com opióides. Estes resultados vêm complementar dados anteriores, que demonstraram que o Plano Nacional Contra as Drogas e as Toxicodependências 2005 - 2012 foi genericamente bem-sucedido, apesar de apresentar áreas passíveis de melhoria no futuro.

\section{CONFLITO DE INTERESSES}

O autor declara que não houve conflito de interesses na realização deste trabalho.

\section{FONTES DE FINANCIAMENTO}

O projecto ACCESS foi patrocinado pela Reckitt Benckiser Pharmaceuticals. A escrita deste artigo teve a assistência da Real Science Communications, UK, financiada pela Reckitt Benckiser.

(update as of mid-2011). [Consultado 2011 Nov 14]. Disponível em http://www.un.org/en/development/desa/policy/wesp/index.shtml.

7. Collins GB, McAllister MS. Buprenorphine maintenance: a new treatment for opioid dependence. Cleve Clin J Med. 2007;74:514-20.

8. Bacha J, Reast S, Pearlstone A. Treatment practices and perceived challenges for European physicians treating opioid dependence. Heroin Addict Relat Clin Probl. 2010;12:9-19.

9. Gjersing L, Waal H, Caplehorn JR, Gossop M, Clausen T. Staff attitudes and the associations with treatment organization, clinical practices and outcomes in opioid maintenance treatment. BMC Health Serv Res. 2010;10:194

10. Greenwald G. Drug decriminalization in Portugal. 2009. [Consultado 2011 Nov 1]. Disponível em http://www.cato.org.

11. Kleber HD. Pharmacologic treatments for opioid dependence: detoxification and maintenance options. Dialogues Clin Neurosci. 2007;9:45570.

12. Stöver H. Barriers to opioid substitution treatment access, entry and retention: a survey of opioid users, patients in treatment, and treating and 
non-treating physicians. Eur Addict Res. 2011;17:44-54.

13. The European Monitoring Centre for Drugs and Drug Addiction (2011) Situation summary for Portugal - up to date summary of the national drug situation in Portugal (Country overviews). [Consultado $2011 \mathrm{Nov}$ 14]. Disponível em http://www.emcdda.europa.eu/publications/country-overviews/pt.

14. National Institutes of Health and U.S.Department of Health and Human Services. Principles of Drug Addiction Treatment. [Consultado 2011 Out 05]. Disponível em http://www.nida.nih.gov.

15. Department of Health (England) and the devolved administrations. Drug misuse and dependence: UK guidelines on clinical management. London: Department of Health, Scottish Government, Welsh Assembly Government and Northern Ireland Executive; 2007

16. Greenwald M, Johanson CE, Bueller J, Chang Y, Moody DE, Kilbourn $M$, et al. Buprenorphine duration of action: mu-opioid receptor availability and pharmacokinetic and behavioral indices. Biol Psychiatry. 2007;61:101-10.

17. Amass L, Ling W, Freese TE, Reiber C, Annon JJ, Cohen AJ, et al. Bringing buprenorphine-naloxone detoxification to community treatment providers: the NIDA Clinical Trials Network field experience. Am J Addict. 2004;13:S42-66.

18. Leonardi C, Hanna N, Laurenzi P, Fagetti R, IDAC. Group. Multi-centre observational study of buprenorphine use in 32 Italian drug addiction centres. Drug Alcohol Depend. 2008:94:125-32.

19. Kamien JB, Branstetter SA, Amass L. Buprenorphine-naloxone versus methadone maintenance therapy: a randomised double-blind trial with opioid-dependent patients. Heroin Addict Relat Clin Probl. 2008;10:5-18.

20. Greenwald MK, Johanson CE, Moody DE, Woods JH, Kilbourn MR Koeppe RA, et al. Effects of buprenorphine maintenance dose on mu-opioid receptor availability, plasma concentrations, and antagonist blockade in heroin-dependent volunteers. Neuropsychopharmacology. 2003;28:2000-9.

21. Apelt SM. Bundesdeutsche multizentrische Beobachtungsstudie zur Substitutionstherapie mit Dosierautomaten in der Versorgung opioidabhängiger Patienten bei 28 Einrichtungen. Suchtmed. 2010;12:243-53.

22. Bell J, Shanahan M, Mutch C, Rea F, Ryan A, Batey R, et al. A randomized trial of effectiveness and cost-effectiveness of observed versus unobserved administration of buprenorphine-naloxone for heroin dependence. Addiction. 2007;102:1899-907.

23. Fiellin DA, Rosenheck RA, Kosten TR. Office-based treatment for opioid dependence: reaching new patient populations. Am J Psychiatry. 2001;158:1200-4

24. Rosenheck R, Kosten T. Buprenorphine for opiate addiction: potential economic impact. Drug Alcohol Depend. 2001;63:253-62.

25. Stöver H, Michels II. Drug use and opioid substitution treatment for prisoners. Harm Reduct J. 2010;7:17. 